\title{
DIFFERENTIAL DESCENT OBSTRUCTIONS OVER FUNCTION FIELDS
}

\author{
JOSÉ FELIPE VOLOCH \\ (Communicated by Matthew A. Papanikolas)
}

\begin{abstract}
We study a new obstruction to the existence of integral and rational points for algebraic varieties over function fields, the differential descent obstruction. We prove that this is the only obstruction to the existence of integral points in affine varieties in characteristic zero and also, in most cases, for rational points on curves in arbitrary characteristic.
\end{abstract}

\section{INTRODUCTION}

Let $K / k$ be a function field of characteristic $p \geq 0$; that is, the function field of a geometrically integral curve over a field $k$, where $k$ is an arbitrary field of characteristic $p \geq 0$. We choose $t \in K$ such that $K / k(t)$ is finite and separable. We denote by $\delta$ the $k$-derivation $d / d t$ of $K$.

Let $S$ be a non-empty set of primes of $K$; we denote by $\mathcal{O}_{S} \subset K$ the ring of $S$-integers. Let $\mathcal{X}$ be an $\mathcal{O}_{S}$-scheme of finite type with generic fibre $X$ over $K$. For each prime of $K$, let $K_{v}$ be the completion of $K$ at $v$ and let $\mathcal{O}_{v}$ be the ring of integers of $K_{v}$. Notation like $\prod_{v} \ldots$ means that the product is taken over all places of $K$. We also extend $\delta$ to $K_{v}$ in the natural way.

The goal of this note is to describe the set $\mathcal{X}\left(\mathcal{O}_{S}\right)$ inside the adelic space $\prod_{v \notin S} \mathcal{X}\left(\mathcal{O}_{v}\right) \times \prod_{v \in S} X\left(K_{v}\right)$ in terms of local conditions involving the coordinates of points and their derivatives with respect to $t$. We call such conditions differential obstructions.

When $p>0$, Harari and the author gave in [6] a local obstruction for integral points on affine varieties over $K$ from covers given by Artin-Schreier equations. Our first goal here will be to give an analogue of this result for $p=0$ using linear differential equations, Theorem 2.2.

Our second goal is to use this result in characteristic $p=0$ or the main result of [6] in characteristic $p>0$ combined with the first jet construction (recalled below) to give differential obstructions to rational points on curves over function fields of arbitrary characteristic. In addition, we will prove a finiteness statement that will give, in particular, that only finitely many such obstructions are necessary.

\section{Obstructions associated to linear differential torsors}

We want to define an obstruction which is analogous to the descent obstruction in the context of differential obstructions. Throughout this section we assume that the characteristic $p=0$.

Received by the editors October 31, 2012.

2010 Mathematics Subject Classification. Primary 11G35, 14G17.

(c) 2014 American Mathematical Society 
Recall the definition of a descent obstruction. Let $G$ be a $K$-group scheme. Let $Y \rightarrow X$ be an $X$-torsor under $G$. We say that a point $\left(x_{v}\right) \in \prod_{v} X\left(K_{v}\right)$ is unobstructed by $Y$ if the evaluation $[Y]\left(\left(x_{v}\right)\right) \in \prod_{v} H^{1}\left(K_{v}, G\right)$ comes from a global element $a \in H^{1}(K, G)$ by the diagonal map. Here all cohomology sets are relative to fppf topology (if $G$ is smooth, then étale topology can be used as well). Note that obviously every family $\left(x_{v}\right) \in \prod_{v} X\left(K_{v}\right)$ coming from a rational point $x \in X(K)$ is unobstructed. We could use the cohomology theory defined in [5] Ch. VII, but we will take a more lowbrow approach, which is consistent with that of [5]; see e.g. VII.6 corollary 1, there. If $\Lambda$ is a linear differential operator, that is, a polynomial in $\delta$ with coefficients in $K$, define $H^{1}(L, \Lambda)$ as $L / \Lambda L$, for any field $L / K$ with an extension of $\delta$ to a derivation of $L$. One thinks of an equation $\Lambda(z)=a$, for $a \in L$ as a torsor for $\Lambda(z)=0$ and the class of $a \in L / \Lambda L$ as the cohomology class of this torsor. If $F$ is a regular function on a variety $X / K$, we say that a point $\left(x_{v}\right) \in \prod_{v} X\left(K_{v}\right)$ is unobstructed by the torsor $\Lambda(z)=F$ if the class $F\left(x_{v}\right) \in \prod_{v} K_{v} / \Lambda\left(K_{v}\right)$ comes from an element of $K / \Lambda(K)$.

Consider the affine line $\mathbf{A}_{K}^{1}$ over $K$ and its integral model $\mathbf{A}_{\mathcal{O}_{S}}^{1}$. Let $f \in K$. We are interested in linear differential torsors $Y_{f}$ for $\Lambda=\delta$ over $\mathbf{A}_{K}^{1}$ given by the equation

$$
Y_{f}: \delta z=f x, \quad x \in \mathbf{A}^{1} .
$$

Proposition 2.1. Assume that $\left(x_{v}\right) \in \prod_{v \notin S} \mathbf{A}^{1}\left(\mathcal{O}_{v}\right) \times \prod_{v \in S} \mathbf{A}^{1}\left(K_{v}\right)$ is unobstructed by $Y_{f}$ for every $f \in K$. Then $\left(x_{v}\right) \in \mathbf{A}^{1}\left(\mathcal{O}_{S}\right)$.

Proof. Let $\omega \neq 0$ be a differential form of $K$ which we write as $\omega=f d t$, where $f \in K$ and $t$ is a separating variable of $K$. Let $x$ be a coordinate on $\mathbf{A}^{1}$ and consider the torsor $Y_{f}$. The fact that $\left(x_{v}\right)$ is unobstructed means that there is an $a \in K$ and for all $v$ there exists $y_{v} \in K_{v}, \delta y_{v}=x_{v} f+a$. It follows that $\operatorname{Res}_{v}\left(x_{v} \omega+a d t\right)=$ $\operatorname{Res}_{v}\left(\delta y_{v}\right) d t=\operatorname{Res}_{v}\left(d y_{v}\right)=0$. Thus, $\sum_{v} \operatorname{Res}_{v}\left(x_{v} \omega\right)=-\sum_{v} \operatorname{Res}_{v} a d t=0$. This being true for all $\omega$ implies that $\left(x_{v}\right)$ is global, by the Riemann-Roch theorem. This completes the proof.

We deduce from the previous proposition the following theorem:

Theorem 2.2. Let $\mathcal{X}$ be an affine $\mathcal{O}_{S}$-scheme of finite type with generic fibre $X$. Let $\left(x_{v}\right) \in \prod_{v \notin S} \mathcal{X}\left(\mathcal{O}_{v}\right) \times \prod_{v \in S} X\left(K_{v}\right)$. Assume that $\left(x_{v}\right)$ is unobstructed by every linear differential torsor $Y \rightarrow X$. Then $\left(x_{v}\right) \in \mathcal{X}\left(\mathcal{O}_{S}\right)$.

Proof. Embed $\mathcal{X}$ into the affine space $\mathbf{A}_{\mathcal{O}_{S}}^{n}$ for some positive integer $n$. Let $\left(\gamma_{v}\right) \in$ $\prod_{v} \mathbf{A}^{1}\left(K_{v}\right)$ be any coordinate of the image of $\left(x_{v}\right)$ in $\prod_{v} \mathbf{A}^{n}\left(K_{v}\right)$. By functoriality $\left(x_{v}\right)$ is unobstructed by every linear differential torsor over $\mathbf{A}^{1}$. By Proposition 2.1. the family $\left(\gamma_{v}\right)$ comes from a global element $\gamma \in \mathbf{A}^{1}\left(\mathcal{O}_{S}\right)$. Since this is true for each coordinate, we obtain that $\left(x_{v}\right) \in \mathcal{X}\left(\mathcal{O}_{S}\right)$. This completes the proof.

Here is an example, completely parallel to one in [6], where a linear differential obstruction is enough to describe integral points.

Proposition 2.3. Let $A, B \in k[t]$ with $\operatorname{deg} A=3, \operatorname{deg} B=1$. Consider the affine scheme over $\mathcal{O}_{S}=k[t]$,

$$
\mathcal{X}: x^{2}+A y^{2}=B .
$$

Assume that $A, B$ are chosen so that the generic fibre $X$ of $\mathcal{X}$ (defined over $K=$ $k(t))$ has a K-point. Then every point in $\prod_{v \neq \infty} \mathcal{X}\left(\mathcal{O}_{v}\right) \times X\left(K_{\infty}\right)$ is obstructed by the torsor $Y: \delta z=y$. In particular $\mathcal{X}$ has no $k[t]$-point. 
Proof. Suppose that there exists a global twist $\delta z=y+c$ with local points everywhere. That is, there exists $z_{v} \in K_{v}, \delta z_{v}=y_{v}+c$, for all $v$. The conditions at $v \neq \infty$ give that the polar parts of $c$ at finite places are integrable (as $y_{v} \in \mathcal{O}_{v}$ and $p=0$ ), so we change $z_{v}$ and assume, without loss of generality, that $c$ is a polynomial. Looking at the equation for $\mathcal{X}$, we see that the component $y_{\infty}$ has valuation equal to 1 at infinity and hence a term in $t^{-1}$, but neither $\delta z_{\infty}$ nor $c$ can have such a term, a contradiction. This completes the proof.

\section{Projective Curves}

Theorem 3.1. Let $X / K$ be a smooth projective curve over a function field $K / k$, with $k$ finite if $k$ has positive characteristic. Assume that $X / K$ has non-zero Kodaira-Spencer class. Then $X(K)$ is described by differential descent obstructions.

Proof. In order to prove the theorem we use a construction from [1]. For any $K$-scheme $X$ one defines the "first jet scheme along $\delta$ " as

$$
X^{1}:=\operatorname{Spec}\left(S\left(\Omega_{X / k}\right) / I\right),
$$

where $I$ is the ideal generated by sections of the form $d f-\delta f\left(f \in \mathcal{O}_{X}\right)$. This object was analyzed in [2,3] in characteristic zero and in [4 in positive characteristic. If $X$ is a smooth variety over $K$, then $\left(\left[1\right.\right.$, p. 1396) $X^{1}$ identifies with the torsor for the tangent bundle $T X:=\operatorname{Spec}\left(S\left(\Omega_{X / K}\right)\right)$ corresponding to the Kodaira Spencer class

$$
\rho(\delta) \in H^{1}\left(X, T_{X / K}\right)
$$

(where $\rho: \operatorname{Der}_{k} K \rightarrow H^{1}\left(X, T_{X / K}\right)$ is the Kodaira Spencer map). We have the following result ([3] if $p=0$ and [4] if $p>0$ ).

Lemma 3.2. If $X / K$ is a smooth projective curve of genus $\geq 2$ with non-zero Kodaira Spencer class, then $X^{1}$ is an affine surface.

We fix an embedding $X^{1} \subset \mathbf{A}^{n}$ with coordinates $x_{1}, \ldots, x_{n}$. Since $\delta$ lifts to a derivation of $K_{v}$, there is a "lifting map"

$$
\nabla: X\left(K_{v}\right) \rightarrow X^{1}\left(K_{v}\right)
$$

We will prove that $\nabla\left(X\left(K_{v}\right)\right)$ is a bounded subset of $\mathbf{A}^{n}\left(K_{v}\right)$ and that, for all but finitely many places $v, \nabla\left(X\left(K_{v}\right)\right) \subset X^{1}\left(\mathcal{O}_{v}\right)$; therefore we get a map of adelic points $\nabla: X\left(A_{K}\right) \rightarrow X^{1}\left(A_{K}\right)$, where $A_{K}$ are the adeles of $K$. First of all, $\nabla$ is continuous for the $v$-adic topology. This will be made clear in the course of the argument. Without loss of generality, we can extend $K$ so that $X(K) \neq \emptyset$, and we fix $P \in X(K)$. If $Q \in X\left(K_{v}\right)$ is $v$-adically sufficiently close to $P$, then $\min \left\{v\left(x_{i}(Q)\right)\right\}=\min \left\{v\left(x_{i}(P)\right)\right\}$. The other points of $X\left(K_{v}\right)$ are $v$-integral points of $U=X-\{P\}$ in a suitable system of coordinates $u_{1}, \ldots, u_{m}$ of an embedding of the affine variety $U$ in $\mathbf{A}^{m}$. If $U$ is cut out by equations $f_{j}=0$, then $U^{1} \subset \mathbf{A}^{2 m}$, and in coordinates $u_{1}, \ldots, u_{m}, v_{1}, \ldots, v_{m}$ is cut out by the equations $\sum_{i} v_{i} \partial f_{j} / \partial u_{i}+$ $f_{j}^{\delta}=0$, where $f_{j}^{\delta}$ is the polynomial obtained by applying $\delta$ to the coefficients of $f_{j}$. Moreover,

$$
\nabla\left(u_{1}, \ldots, u_{m}\right)=\left(u_{1}, \ldots, u_{m}, \delta u_{1}, \ldots, \delta u_{m}\right)
$$

(so the continuity of $\nabla$ is clear). It is also clear that, for integral points $Q$ of $U\left(K_{v}\right)$, the coordinates of $\nabla(Q) \in \mathbf{A}^{2 m}$ are bounded and the same holds for the coordinates in $\mathbf{A}^{n}$. Finally, if $S$ is a sufficiently large set of places of $K$ for which 
all the constant elements of $K$ appearing in the above argument are $S$-units, then for $v \notin S, \nabla\left(X\left(K_{v}\right)\right) \subset X^{1}\left(\mathcal{O}_{v}\right)$.

We are then all set to apply Theorem 2.2, if $p=0$, or the main result of [6], if $p>0$, to complete the proof.

We remark that, since we showed that $\nabla\left(X\left(K_{v}\right)\right) \subset X^{1}\left(K_{v}\right)$ is bounded for all $v$, one only needs to use finitely many differential forms as in the proof of Theorem 2.2 if $p=0$, or the main result of [6], if $p>0$, to obstruct the points, leading to an algorithm to decide whether or not $X(K)$ is empty. A local-global obstruction for curves over function fields was previously obtained in [7] but, in positive characteristic, the hypotheses there are different from the ones here, so there are cases where one result can be applied when the other cannot.

\section{ACKNOWLEDGEMENTS}

The author would like to thank the Simons Foundation (grant \#234591) and the Centre Bernoulli at EPFL for financial support.

\section{REFERENCES}

[1] A. Buium, Intersections in jet spaces and a conjecture of S. Lang, Ann. of Math. (2) 136 (1992), no. 3, 557-567, DOI 10.2307/2946600. MR1189865(93j:14055)

[2] Alexandru Buium, Geometry of differential polynomial functions. I. Algebraic groups, Amer. J. Math. 115 (1993), no. 6, 1385-1444, DOI 10.2307/2374970. MR1254738 (95c:12011)

[3] Alexandru Buium, Geometry of differential polynomial functions. II. Algebraic curves, Amer. J. Math. 116 (1994), no. 4, 785-818, DOI 10.2307/2375002. MR.1287940 (96a:14039)

[4] Alexandru Buium and José Felipe Voloch, Lang's conjecture in characteristic p: an explicit bound, Compositio Math. 103 (1996), no. 1, 1-6. MR1404995 (98a:14038)

[5] E. R. Kolchin, Differential algebraic groups, Pure and Applied Mathematics, 114, Academic Press, Inc., Orlando, FL, 1985. MR0776230 (87i:12016)

[6] D. Harari and J. F. Voloch, Descent obstructions and Brauer-Manin obstruction in positive characteristic, J. Inst. Math. Jussieu 12 (2013), no. 3, 545-551. MR3062870

[7] Bjorn Poonen and José Felipe Voloch, The Brauer-Manin obstruction for subvarieties of abelian varieties over function fields, Ann. of Math. (2) 171 (2010), no. 1, 511-532, DOI 10.4007/annals.2010.171.511. MR2630046 (2011j:14048)

Department of Mathematics, University of Texas, Austin, Texas 78712

E-mail address: voloch@math.utexas.edu 